\title{
Dynamic fracture effects observed in a one- dimensional discrete mechanical system
}

\author{
Nikita Kazarinov ${ }^{1,2^{*}}$, Alexander Smirnov ${ }^{2}$, Yuri Petrov $^{2,4}$, Alexey Gruzdkov ${ }^{3}$ \\ ${ }^{1}$ Emperor Alexander I Saint Petersburg State Transport University, 9, Moskovsky av., Saint \\ Petersburg, 190031, Russia \\ ${ }^{2}$ Saint Petersburg State University, 7-9, Universitetskaya Emb., Saint Petersburg, 199034, Russia \\ ${ }^{3}$ Institute of Problems of Mechanical Engineering (RAS), 61 Bolshoi av. VO, Saint Petersburg, \\ 199178, Russia \\ ${ }^{4}$ Saint Petersburg State University of Technology, 26 Moskovsky av., Saint Petersburg, 190013, \\ Russia
}

\begin{abstract}
Dynamic fracture of a one-dimensional chain of identical linear oscillators (masses connected by springs) is considered in the work. The system is supposed to consist of arbitrary but finite number of links and the first mass is supposed to be fixed. Two loading conditions are discussed: free oscillations of an initially statically prestressed chain and loading the chain with a short deformation pulse. Both problems are solved analytically for an arbitrary number of links. The obtained solutions are investigated and a dynamic fracture effect related to an explicitly discrete structure of the system is revealed: a deformation wave travelling through the chain is distorted and some links may be subjected to critical deformation. The obtained solutions for the chain are compared to the solutions of analogous problems stated for an elastic rod - a continuum counterpart of the considered discrete system. It is shown that the discussed fracture effect cannot be found in a continuous system.
\end{abstract}

\section{Introduction}

Mass-spring models are a common tool in mechanics due to their simplicity and ability to address rather complicated phenomena. For example, in work [1] the oscillator model is coupled with finite element method to address acoustic emission studies of rocks.

Oscillator chains were considered in works by L. Slepyan and his co-workers [2], however this approach considers infinite oscillator chains. Moreover, the chain models are successfully used to study peculiar heat conduction effects in crystals. Two-dimensional models have been also used to address effects encountered in dynamic crack propagation problems. For example, in work [3] the crack velocity oscillations are explained using a lattice model, while in [4] a bi-material model is studied in order to investigate various regimes of the interface crack propagation. The chain models have been also used to address martensitic phase transformations as seen from work [5].

\footnotetext{
${ }^{*}$ Corresponding author: n.kazarinov@spbu.ru
} 
Simple mass-spring models have been effectively applied to study rate sensitivity of materials and inverse rate sensitivity in particular [6].

In this paper dynamic fracture effects related to discreetness of the oscillator chain system are discussed. Firstly, analytic solution for the system of differential equations governing the chain movement is obtained. This solution is then compared to the solution of a onedimensional wave equation, which describes wave propagation in an elastic rod - a continuous analogue of the oscillator chain.

\section{Static preload with abrupt link failure}

\subsection{Analytic solution of the chain problem}

Consider a uniformly deformed chain consisting of $N+1$ equal linear oscillators with both ends fixed.

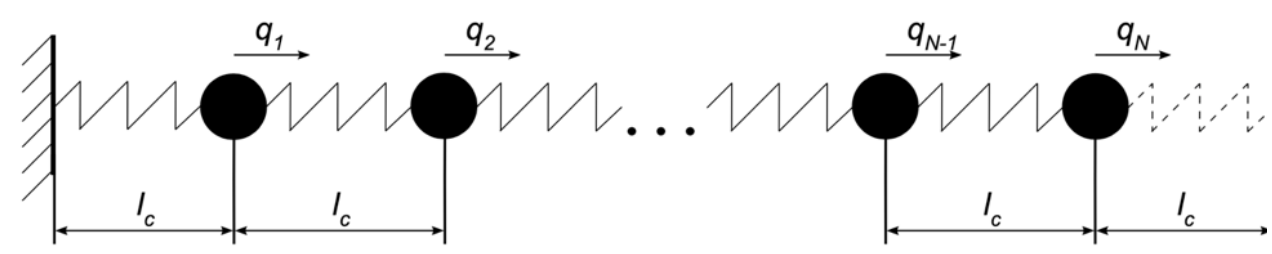

Fig. 1. Uniformly deformed chain with an abruptly failing link.

If the masses are taken equal $m$, stiffnesses of the springs $-c$, the following system of differential equations coupled with initial conditions describes the chain motion:

$$
\begin{aligned}
& \boldsymbol{M} \ddot{\boldsymbol{Q}}+\boldsymbol{C Q}=\mathbf{0} \\
& q_{i}(t=0)-q_{i-1}(t \\
& =0 \text { ) } \\
& =l_{c} \\
& \dot{q}_{l}(t=0)=0 \\
& q_{0}(t)=0
\end{aligned}
$$

where $\boldsymbol{Q}=\left(q_{1}, q_{2}, . ., q_{N}\right)$ is a vector containing relative mass displacements, $\boldsymbol{M}$ is the mass matrix and $\boldsymbol{C}$ is a stiffness matrix and $l_{c}$ is critical link deformation. Moreover, it is supposed that link with number $N+1$ (dashed link in figure 1) does not bear this load and breaks abruptly at $t=0$ initiating a release wave. The following fracture conditions are used: $\left|q_{i}-q_{i-1}\right|>l_{c}, i=1 . . N$. Matrices $\boldsymbol{M}$ and $\boldsymbol{C}$ read as:

$\boldsymbol{M}=m \boldsymbol{E}, \boldsymbol{E}$ is identity matrix

$$
\boldsymbol{C}=c\left(\begin{array}{ccccc}
2 & -1 & \cdots & 0 & 0 \\
-1 & 2 & -1 & \cdots & 0 \\
& \ddots & \ddots & \ddots & \\
0 & & -1 & 2 & -1 \\
0 & 0 & \cdots & -1 & 1
\end{array}\right)=c \boldsymbol{K}
$$

One of the chain ends remains fixed, and therefore $q_{0}(t)=0 \forall t$. The substitution $t^{\prime}=$ $t \sqrt{c / m}$ yields the dimensionless problem with the mass matrix equaling identity matrix and the stiffness matrix $\boldsymbol{K}=\frac{1}{c} \boldsymbol{C}$ from (2). Additionally, normalized deformations of the chain 
links are introduced according to equation $u_{i}=q_{i}-q_{i-1} / l_{c}, i=1 . . N$ resulting in a modified stiffness matrix $\boldsymbol{K}=\boldsymbol{S} \boldsymbol{K}^{\prime} \boldsymbol{S}^{-1}$ with $S$ being a matrix of coordinates transformation. The fracture condition has the form: $\left|u_{i}(t)\right|>1, i=1 . . N$. This way, the following problem is solved if vector $\boldsymbol{U}=\left(u_{1}, u_{2}, \ldots, u_{N}\right)$ is introduced:

$$
\begin{gathered}
\ddot{\boldsymbol{U}}+\boldsymbol{K} \boldsymbol{U}=\mathbf{0} \\
u_{i}(t=0)=1 \\
\dot{u}_{\imath}(t=0)=0 \\
u_{0}(t)=0 \\
\left|u_{i}(t)\right|>1, i=1 . . N
\end{gathered}
$$

Solution of the system (3) is sought in form

$$
\boldsymbol{U}(\boldsymbol{t})=\sum_{j=1}^{n} c_{j} \boldsymbol{R}_{j} \cos \left(\omega_{j} t\right)
$$

where $\omega_{j}$ are the system eigenfrequencies, $\boldsymbol{R}_{\boldsymbol{j}}=\left(r_{1}^{(j)}, r_{2}^{(j)}, \ldots, r_{N}^{(j)}\right)^{\boldsymbol{T}}$ are corresponding eigenvectors and $c_{j}$ is the set constants evaluated using the initial conditions.

The eigenfrequencies are calculated from eigenvalues $\lambda_{j}$ of the system stiffness matrix $\boldsymbol{K}: \omega_{j}=\sqrt{\lambda_{j}}$. The eigenvalues of $\boldsymbol{K}$ are calculated from equation

$$
\begin{aligned}
\operatorname{Det}(\boldsymbol{K}-\lambda \boldsymbol{E}) & =\operatorname{Det}\left(\boldsymbol{K}^{\prime}\right. \\
& -\lambda \boldsymbol{E})=0
\end{aligned}
$$

If we put $\alpha=2-\lambda$, (5) can be rewritten explicitly in the following way:

$$
\left|\begin{array}{ccccc}
\alpha & -1 & \cdots & 0 & 0 \\
-1 & \alpha & -1 & \cdots & 0 \\
& \ddots & \ddots & \ddots & \\
0 & & -1 & \alpha & -1 \\
0 & 0 & \cdots & -1 & \alpha-1
\end{array}\right|=D_{N}=0
$$

In (6) $D_{N}$ is determinant of order $N$. One may note that a recursive equation can be composed for a determinant of order $k$ :

$$
D_{k}=\alpha D_{k-1}-D_{k-2}
$$

and the following relations hold: $D_{0}=1, D_{1}=\alpha-1$. The equation (7) is reduced to a quadratic equation $p^{2}-\alpha p+1=0$ with roots $p_{1,2}$ using substitution $D_{k}=p^{k}$. This way the following expression is obtained from (6):

$$
D_{N}=b_{1} p_{1}^{N}+b_{2} p_{2}^{N}=0
$$

Where $b_{1}$ and $b_{2}$ are constants to be evaluated using conditions $D_{0}=1, D_{1}=\alpha-1$ and substitution $\alpha=2 \cos (\theta)$. Equation (8) yields the following formula for eigenvalues of the stiffness matrix:

$$
\lambda_{k}=2-2 \cos \left(\frac{\pi(2 k-1)}{2 N+1}\right), k=1 . . N
$$

and thus, we obtain the formula for eigenfrequencies of the studied system: 


$$
\begin{gathered}
\omega_{k}=2 \sin \left(\frac{\pi(2 k-1)}{4 N+2}\right), k \\
=1 . . N
\end{gathered}
$$

For components of the eigenvectors of matrix $\boldsymbol{K}$ the following equation holds:

$$
r_{i}^{j}=P_{i-1}\left(x_{j}\right)-P_{i-2}\left(x_{j}\right)
$$

where $x_{j}=\left(2-\lambda_{j}\right) / 2$ and $P_{k}(x)$ is a k-order Chebyshev polynomial of second kind, which can be expressed in the following way:

$$
\begin{aligned}
& P_{k}(y) \\
& =\frac{\sin ((k+1) \arccos (y))}{\sin (\arccos (y))}
\end{aligned}
$$

Thus, the following expression can be obtained for the components of eigenvectors $\boldsymbol{R}_{\boldsymbol{j}}$ :

$$
r_{i}^{j}=\frac{\cos \left(\frac{\pi(2 i-1)(2 j-1)}{4 N+2}\right)}{\cos \left(\frac{\pi(2 j-1)}{4 N+2}\right)}, i=1 . . N
$$

In order to evaluate constants $c_{j}$ to satisfy the initial conditions the following system should be solved accounting for (11):

$$
\begin{gathered}
\left(\begin{array}{cccc}
P_{0}\left(x_{1}\right) & P_{0}\left(x_{2}\right) & \cdots & P_{0}\left(x_{N}\right) \\
P_{1}\left(x_{1}\right)-P_{0}\left(x_{1}\right) & P_{1}\left(x_{2}\right)-P_{0}\left(x_{2}\right) & \cdots & P_{1}\left(x_{N}\right)-P_{0}\left(x_{N}\right) \\
\vdots & \vdots & \vdots & \vdots \\
P_{N-1}\left(x_{1}\right)-P_{N-2}\left(x_{1}\right) & P_{N-1}\left(x_{2}\right)-P_{N-2}\left(x_{2}\right) & \cdots & P_{N-1}\left(x_{N}\right)-P_{N-2}\left(x_{N}\right)
\end{array}\right)\left(\begin{array}{c}
c_{1} \\
c_{2} \\
\vdots \\
c_{N}
\end{array}\right)= \\
=\left(\begin{array}{c}
1 \\
1 \\
\vdots \\
1
\end{array}\right)=\left(\begin{array}{c}
P_{0}(1) \\
P_{1}(1)-P_{0}(1) \\
\vdots \\
P_{N-1}(1)-P_{N-2}(1)
\end{array}\right)
\end{gathered}
$$

If the second order Chebyshev polynomials are explicitly written and elementary matrix operations are performed, the system (14) is reduced to the system with a Vandermonde matrix:

$$
\left(\begin{array}{cccc}
1 & 1 & \cdots & 1 \\
x_{1} & x_{2} & \cdots & x_{N} \\
\vdots & \vdots & \vdots & \vdots \\
x_{1}{ }^{N-1} & x_{2}{ }^{N-1} & \cdots & x_{N}{ }^{N-1}
\end{array}\right)\left(\begin{array}{c}
c_{1} \\
c_{2} \\
\vdots \\
c_{N}
\end{array}\right)=\left(\begin{array}{c}
1 \\
1 \\
\vdots \\
1
\end{array}\right)
$$

Constants $c_{j}$ can be evaluated from (15) using Cramer rule and formula for the determinant of the Vandemonde matrix:

$$
c_{j}=\frac{\left(1-x_{1}\right) \cdots\left(1-x_{j-1}\right)\left(1-x_{j+1}\right) \cdots\left(1-x_{N}\right)}{\left(x_{j}-x_{1}\right) \cdots\left(x_{j}-x_{j-1}\right)\left(x_{j}-x_{j+1}\right) \cdots\left(x_{j}-x_{N}\right)}
$$

Let's put $M_{N}(x)=2^{N} \prod_{k=1}^{N}\left(x-x_{k}\right)$. Then (16) can be rewritten: 


$$
c_{j}=\frac{M_{N}(1)}{M_{N}{ }^{\prime}\left(x_{j}\right)\left(1-x_{j}\right)}
$$

Considering the fact that $M_{N}(x)$ has zeros at points $x_{j}$ and multiplier $2^{N}$, one may conclude that $M_{N}(x)=P_{N}(x)-P_{N-1}(x)$ and thus one can deduce formula for constants $c_{j}$ taking into account that $M_{N}(1)=1$ :

$$
c_{j}=\frac{(-1)^{j+1} 2 \sin \left(\frac{\pi(2 j-1)}{2 n+1}\right) \cos \left(\frac{\pi(2 j-1)}{4 n+2}\right)}{(2 n+1)\left(1-\cos \left(\frac{\pi(2 j-1)}{2 n+1}\right)\right)}
$$

Now general solution of the problem (3) is the following:

$$
\begin{gathered}
u_{i}(t)=\frac{2}{(2 N+1)} \sum_{j=1}^{n} \frac{(-1)^{j+1} \sin \left(\beta_{j}\right) \cos \left(\frac{\beta_{j}}{2}(2 i-1)\right)}{\left(1-\cos \left(\beta_{j}\right)\right)} \cos \left(\omega_{j} t\right), \\
\beta_{j}=\frac{\pi(2 j-1)}{2 N+1}
\end{gathered}
$$

In (19) $u_{i}(t)$ stands for deformation of the chain link with number $i$.

\subsection{Forced chain oscillations, inhomogeneous system of equations.}

The following problem is considered: a chain of oscillators with $N$ links and a fixed end is loaded with an arbitrary force $f(t)$ applied to the chain free end (Fig. 2).

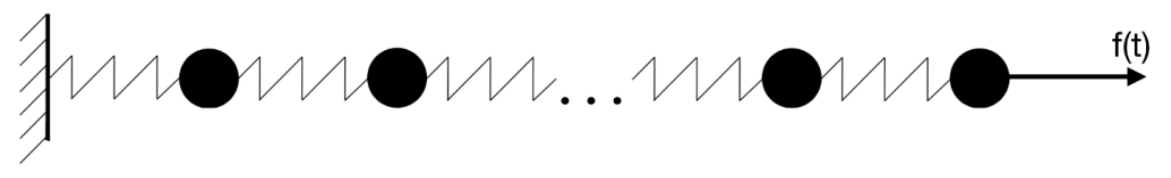

Fig. 2. Chain loaded with an arbitrary force $f(t)$.

The system of dimensionless equations describing deformation of the chain links is the following:

$$
\begin{aligned}
& \ddot{\boldsymbol{U}}+\boldsymbol{K} \boldsymbol{U}=\boldsymbol{F}(\boldsymbol{t})=(0,0, \ldots, f(t))^{T} \\
& \begin{array}{l}
u_{i}(t=0)=0 \\
\dot{u}_{\imath}(t=0)=0
\end{array} i=1 . . N \\
& u_{0}(t)=0
\end{aligned}
$$

In order to solve system (20) an auxiliary homogeneous system of differential equations with modified initial conditions is introduced and solved following Duhamel's method (system inhomogeneity is transferred to the initial conditions [7]:

$$
\begin{aligned}
& \ddot{\boldsymbol{W}}+\boldsymbol{K} \boldsymbol{W}=\mathbf{0} \\
& w_{i}(t=0)=0, i=1 . . N \\
& \dot{w}_{l}(t=0)=0, i=1 . . N-1
\end{aligned}
$$




$$
\begin{aligned}
& \dot{w}_{N}(t=0)=f(p) \\
& w_{0}(t)=0
\end{aligned}
$$

where $p$ is an arbitrary real number. Systems (21) and (22) share stiffness matrix $\boldsymbol{K}$ with the system solved in the previous section. Solution of (21) is further obtained as a convolution of solution of (22).

Solution steps for (22) are similar to those for (3). The general solution is sought in form

$$
\boldsymbol{W}(\boldsymbol{t})=\sum_{j=1}^{N} a_{j} \boldsymbol{R}_{j} \sin \left(\omega_{j} t\right)
$$

where eigenfrequencies and eigenvectors $\omega_{j}$ and $\boldsymbol{R}_{\boldsymbol{j}}$ are evaluated according to formulas (10) and (13). In order to obtain the solution constants $a_{j}$ should be calculated satisfying the initial conditions. Let's put $b_{j}=a_{j} \omega_{j}$. Then the system for $b_{j}$ reads as:

$$
\left(\begin{array}{cccc}
P_{0}\left(x_{1}\right) & P_{0}\left(x_{2}\right) & \cdots & P_{0}\left(x_{N}\right) \\
P_{1}\left(x_{1}\right)-P_{0}\left(x_{1}\right) & P_{1}\left(x_{2}\right)-P_{0}\left(x_{2}\right) & \cdots & P_{1}\left(x_{N}\right)-P_{0}\left(x_{N}\right) \\
\vdots & \vdots & \vdots & \vdots \\
P_{N-1}\left(x_{1}\right)-P_{N-2}\left(x_{1}\right) & P_{N-1}\left(x_{2}\right)-P_{N-2}\left(x_{2}\right) & \cdots & P_{N-1}\left(x_{n}\right)-P_{N-2}\left(x_{N}\right)
\end{array}\right)\left(\begin{array}{c}
b_{1} \\
b_{2} \\
\vdots \\
b_{N}
\end{array}\right)=\left(\begin{array}{c}
0 \\
0 \\
\vdots \\
f(p)
\end{array}\right)
$$

In (24) $x_{j}=\left(2-\lambda_{j}\right) / 2$ and $P_{k}(x)$ is a k-order Chebyshev polynomial of second kind. As in the previous case (24) is reduced to a system with a Vandermonde matrix:

$$
\left(\begin{array}{cccc}
1 & 1 & \cdots & 1 \\
x_{1} & x_{2} & \cdots & x_{N} \\
\vdots & \vdots & \vdots & \vdots \\
x_{1}{ }^{N-1} & x_{2}{ }^{N-1} & \cdots & x_{N}{ }^{N-1}
\end{array}\right)\left(\begin{array}{c}
b_{1} \\
b_{2} \\
\vdots \\
b_{N}
\end{array}\right)=\left(\begin{array}{c}
0 \\
0 \\
\vdots \\
f(p) / 2^{N-1}
\end{array}\right)
$$

If the Cramer's rule is applied and function $M_{N}(x)=2^{N} \prod_{k=1}^{N}\left(x-x_{k}\right)$ is introduced, the following formula holds:

$$
b_{j}=\frac{2 f(t)}{M_{N}^{\prime}\left(x_{j}\right)}
$$

Thus, if $M_{N}(x)$ is expressed using $P_{k}(x)$, final formula for the constants $a_{j}$ can be written:

$$
b_{j}=\frac{(-1)^{j+1} 4 \cos ^{2}\left(\frac{\pi(2 j-1)}{4 N+2}\right) f(t)}{(2 N+1)}
$$

Since the auxiliary system is explicitly solved, solution to the initial system (21) can be written:

$$
\boldsymbol{U}(\boldsymbol{t})=\sum_{j=1}^{N} a_{j} \boldsymbol{R}_{\boldsymbol{j}} \int_{0}^{t} \sin \left(\omega_{j}(t-s)\right) f(s) d s
$$

The following loading function $f(t)$ is considered if pulse load is studied: 


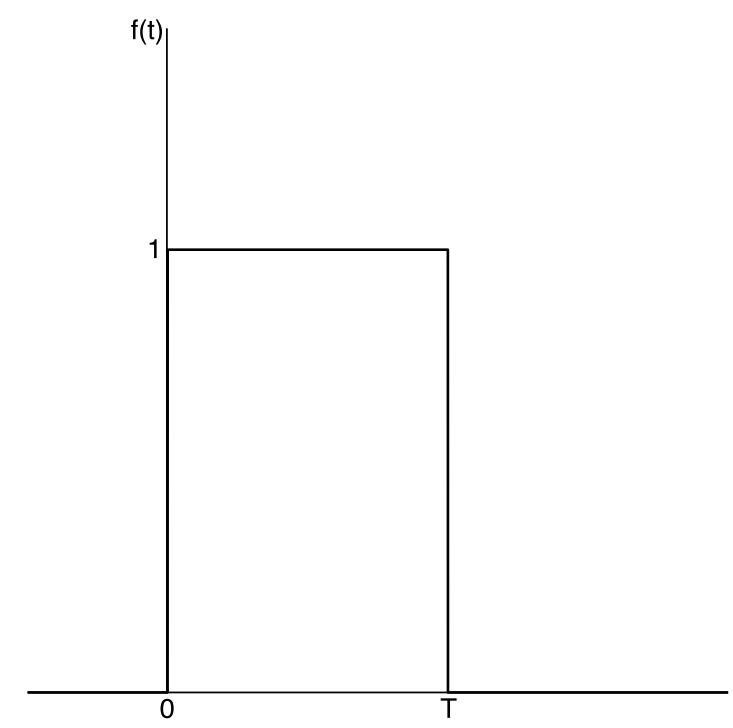

Fig.3. Loading function: pulse with duration $T$.

Then (28) is transformed into the following expression:

$$
\boldsymbol{U}(\boldsymbol{t})=\left\{\begin{array}{c}
\sum_{j=1}^{N} \frac{a_{j} \boldsymbol{R}_{\boldsymbol{j}}}{\omega_{j}}\left(1-\cos \left(\omega_{j} t\right)\right), t<T \\
\sum_{j=1}^{N} \frac{a_{j} \boldsymbol{R}_{\boldsymbol{j}}}{\omega_{j}}\left(\cos \left(\omega_{j}(t-T)\right)-\cos \left(\omega_{j} t\right)\right), t \geq T
\end{array}\right.
$$

Thus, (29) is a formula for deformation of links of the chain subjected to pulse load.

\section{Comparison with solutions for an elastic rod.}

In this section formulas (19) and (29) will be used to evaluate deformations in particular chain links. Moreover, these solutions will be compared to deformations of an elastic rod, subjected to similar loads. The rod can be regarded as a continuous counterpart of the chain.

A prestressed elastic rod is a complete analogue of the chain problem considered in 2.1. A release wave propagation in the homogenously deformed elastic rod of length $l$ with model material parameters (elastic modulus and density equal 1) is considered. If displacements of the rod points are described by function $U(x, t)$ and deformations by $\varepsilon(x, t)$ and sealing of the rod end $x=0$ is supposed, the following initial boundary value problem can be stated: 


$$
\begin{gathered}
\frac{\partial^{2} U(x, t)}{\partial x^{2}}=\frac{\partial^{2} U(x, t)}{\partial t^{2}} \\
U(x, 0)=x \Rightarrow \varepsilon(x, t)=1 \\
\left.\frac{\partial U(x, t)}{\partial t}\right|_{t=0}=0 \\
U(0, t)=0 \\
\left.\frac{\partial U(x, t)}{\partial x}\right|_{x=l}=H(-t)
\end{gathered}
$$

Additionally, the following fracture condition is set: fracture takes place if $|\varepsilon(x, t)|>1$. Solution of (30) can be obtained as a combination of travelling and reflected waves. In figure 4 deformation of the first link and deformation of the rod sealing are shown. It is clear that deformations of the elastic rod never exceed the initial value 1 and thus fracture never takes place, while deformation of the first link of the chain exceeds critical value by about $50 \%$ leading to the system failure. This way, equal loading conditions result in fracture for the discrete system, while its continuous analogue remains intact.

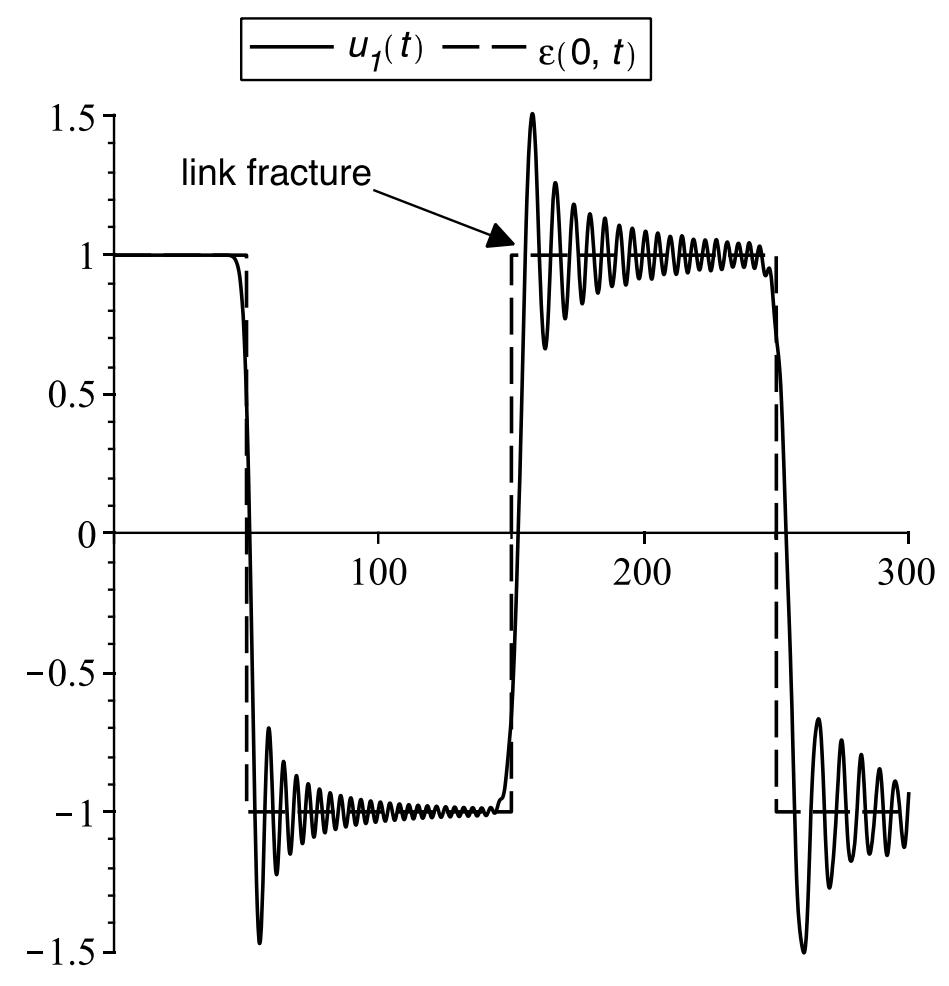

Fig. 4. Deformation of the first chain link (solid line) and deformation of rod in a sealed point (dashed line). Arrow indicates the link fracture. Results for 50 links and a rod with length $l=50$ are shown.

Now the rod is supposed to be loaded by a deformation pulse. Model material is used and the rod is supposed to be sealed from one end. Thus, the initial boundary value problem reads as: 


$$
\begin{gathered}
\frac{\partial^{2} \varepsilon(x, t)}{\partial x^{2}}=\frac{\partial^{2} \varepsilon(x, t)}{\partial t^{2}} \\
\varepsilon(x, 0)=0 \\
\left.\frac{\partial \varepsilon(x, t)}{\partial t}\right|_{t=0}=0 \\
U(0, t)=0 \\
\varepsilon(l, t)=f(t)=H(t)-H(t-T)
\end{gathered}
$$

If d'Alembert method is applied, one can find that an undistorted deformation pulse $f(t)$ travels through the rod and no fracture occurs, since $|\varepsilon(x, t)|>1$ fracture condition is considered. On the contrary, solution for the chain shows distortion of the pulse, which leads to failure of the link with number $N$. This phenomenon is shown in figure 5 for a chain consisting of 100 links, pulse duration $T=10$ and rod with length $l=100$.

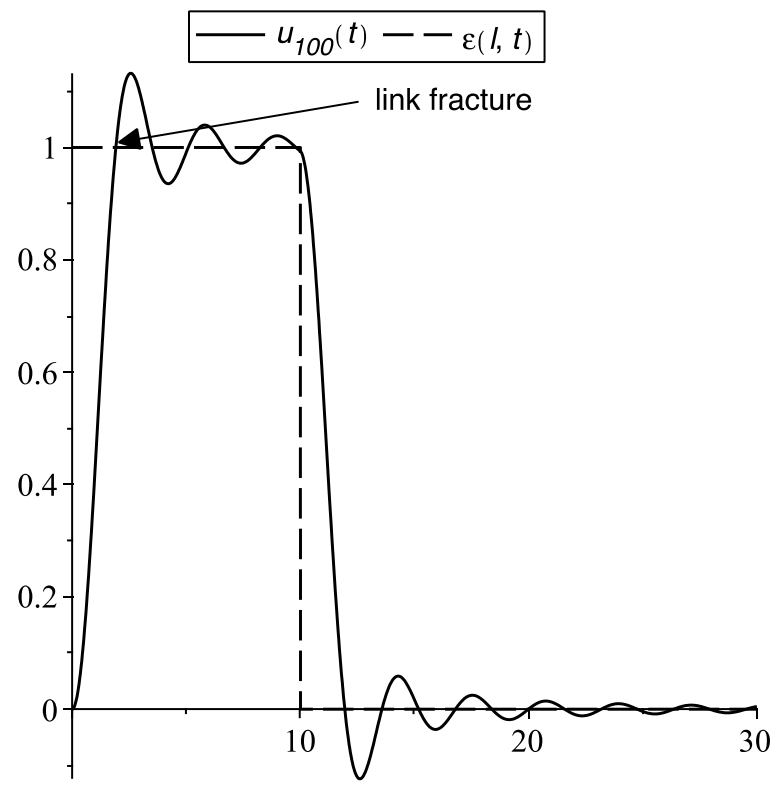

Fig. 5. Deformation of the chain link with number $N$ (solid line) and rod deformation at point $x=l$ (dashed line). Link fracture is indicated by an arrow. In this case number of links $N=100$ and rod length $l=100$.

This way pulse load applied to a discrete system may lead to failure, while continuous system remains intact for the identical load.

\section{Conclusion}

Dynamic fracture of linear oscillator chains is considered in the work. In particular effect related to discreetness of the system is studied. Two load cases are considered: abrupt release of a prestressed chain and pulse loading of an undeformed chain. For the both cases analytical solutions for the chain link deformations are obtained. These solutions are compared to results for a continuous analogue of chain - elastic rod. It is demonstrated that the wave 
travelling through a chain (resulting either from abrupt release or from deformation pulse applied) is distorted comparing to an elastic rod, which can result into fracture. On the contrary, such effect is not possible for the continuous system.

This effect can be accounted for when structures with explicit discreetness and periodicity are designed and studied, e.g. construction facilities in civil engineering. A railway train could serve as another example of possible application of the studied discrete problem. A railway train moving with a constant velocity can be modeled by a statically uniformly deformed chain of oscillators. Thus, a sudden break of a damaged or worn coupling device can potentially lead to failure of normally functioning coupling devices.

The work was supported by Russian Foundation for Basic Research (grants 19-31-60037 and 1851-80008); N. Kazarinov gratefully acknowledges support from Russian Science Foundation (grant 1871-00107) for creation of sections 2.2 and 3 being affiliated with Saint Petersburg State Uni versity

\section{References}

1. D. T. Ngo, F.L. Pellet, Engineering Fracture Mechanics, 224106802 (2020). https://doi.org/10.1016/j.engfracmech.2019.106802

2. L.I. Slepyan, L.V. Troyankina, J. Appl. Mech. Techn. Phys., 25, 921-927 (1984). https://doi.org/10.1007/BF00911671

3. M. Marder, S.Gross, J.Mech.Phys.Solids, 43(1), 1-48 https://doi.org/10.1016/0022-5096(94)00060-I

4. N. Gorbushin, G. Mishuris, Phil. Trans. R. Soc. A, 377, 20190103 (2019). https://doi.org/10.1098/rsta.2019.0103

5. L. Truskinovsky, A. Vainchtein, SIAM J. Appl. Math., 66(2), 533-553 (2005). https://doi.org/10.1137/040616942

6. T.R. McNelley, S.F. Gates, Acta Metallurgica, 26, 1605-1614 (1978). https://doi.org/10.1016/0001-6160(78)90070-6

7. M. Epstein, Partial Differential Equations: Mathematical Techniques for Engineers (Mathematical Engineering) (Springer, 2017) https://doi.org/10.1007/978-3-319-55212$\underline{5}$ 